\title{
Inhalt, Vol. 10, No. 4, 1987
}

\section{Contents}

Impressum 182

Editorial 184

Kurth, R.; Lower, R. und Lower, J., Frankfurt;

Gelderblom, H., Berlin

Retroviren und Lymphomentstehung 186

Hinweise für Autoren 194

Bornkamm, G. W.; Polack, A.; Eick, D.

und Berger, R., Freiburg; Lenoir, G., LyonlFrankreich

Chromosomentranslokationen und Epstein-Barr-Virus beim

Burkitt-Lymphom 196

Fonatsch, Ch. und Gradl, G., Lübeck

Chromosomenaberrationen und maligne Lymphome206

Ehninger, G.; Schuler, 11. und Waller, H.D., Tubingen Pharmakokinetische Aspekte beim

Einsatz neuer Zytostatika . . 218

Gallmeier, W.M. und Bruntsch, U., Nürnberg

Neue Zytostatika-Stand: 1986226

Schwendener, R.A.; Supersaxo, A.; Rubas, W.

und Hengartner, H., Zurich!Schweiz; Schott, H., Tubingen;

Hartmann, H.R., BasellSchweiz

Liposomen als Träger von lipophilen Cytosinarabínosid- und

Fluorodeoxyuridin-Derivaten - Ihre zytostatische Wirkung und

Möglichkeiten zur tumorzellspezifischen Therapie 232

Kalden, J.R., Erlangen

Zytokine in der Tumortherapie

Gall, F. P. , Erlangen

Die Resektion von Lebermetastasen 247

Franke, R.P.; Höpken, S.; Schnittler, H.; Dauer, U.; Zangs, R.

und Mittermayer, Ch., Aachen; Kiesewetter, H., Homburg

Endothelfunktion und Arteriosklerose 250

Nawroth, P.P. und Stern, D.M., Oklahoma City/USA

Veränderung der Gerinnungseigenschaften des Endothels durch

Tumor Nekrosis-Faktor 254

Sonderbände 258

Risau, W, Tubingen

Endothel, Angiogenese und Metastasierung 260

Informationen für die Klinik 263

Zacharski, L.R., Vermont/USA 
Kleinzelliges Bronchialkarzinom: Interaktionen mit dem Gerinnungssystem und Behandlung mit Antikoagulanzien264

Imprint 182

Editorial 184

Kurth, R.; Lower, R. and Lower, J., Frankfurt;

Gelderblom, H., Berlin

Retroviruses and Development of Lymphomas

186

Instructions for Authors 194

Bornkamm, G. W.; Polack, A.; Eick, D.

and Berger, R., Freiburg; Lenoir, G., Lyonl France

Chromosomal Translocations, Epstein-Barr Virus and Burkitt's

Lymphoma 196

Fonatsch, Ch. and Gradl, G., Lübeck

Chromosomal Aberrations and Malignant Lymphomas

Ehninger, G.; Schuler, U. and Waller, H.D., Tubingen

Pharmacokínetic Aspects of Anticancer Treatment 218

Gallmeier, W.M. and Bruntsch, U., Nuremberg

New Cytotoxic Drugs 226

Schwendener, R.A.; Supersaxo, A.; Rubas, W.

and Hengartner, H., Zurich/Switzerland; Schott, H., Tubingen;

Hartmann, H.R., Basel/Switzerland

Liposomes as Carriers for Lipophilic Cytosine Arabinoside and

Fluorodeoxyuridine Prodrugs. - Their Cytostatic Activity and

Possibilities for a Tumor-Cell Specific Therapy 232

Kalden, J.R., Erlangen

Cytokines in Tumor Therapy 240

Gall, F. P., Erlangen

Resection of Liver Metastases

Franke, R.P.; Höpken, S.; Schnittler, H.; Dauer, U.; Zangs, R.

and Mittermayer, Ch., Aachen; Kiesewetter, H., Homburg

Endothelial Function and Arteriosclerosis 250

Nawroth, P.P. and Stern, D.M., Oklahoma City/USA

Tumor Necrosis Factor/Cachectin-Induced Modulation of Endo

thelial Cell Hemostatic Properties 254

Special Editions 258

Risau, W., Tubingen

Endothelium, Angiogenesis and Metastasis 260

Clinical Information 263

Zacharski, L.R., Vermont/USA

Small Cell Carcinoma of the Lung: Interaction with the Blood

Coagulation Mechanism and Treatment with Anticoagulants. . . 264

Teil I wurde in der ONKOLOGIE 3/Juní 1987 publiziert.

Die Beiträge von Teil I+ 11 wurden zusammengestellt von: H.D. Waller und B. Steinke, Med.

Klinik der Universität Tubingen.

Teil I + Teil II sind auch als Sammelband zu beziehen (siehe Seite 259). 
Bibliographischer Hinweis: Inhaltsverzeichnisse dieser Zeitschrift erscheinen regelmäßig in current contents ${ }^{\circledR}$ sowie in anderen bibliographischen Diensten. 